\title{
Dal CRM al social CRM: uno strumento per incrementare le performance della relazione con il cliente

\author{
Roberto Chierici - Niccolò Gordini
}

\begin{abstract}
Obiettivo del paper: Basandosi sulla teoria delle capacità dinamiche lo studio esamina, da un lato, il passaggio dal CRM al social CRM e, dall'altro, l'impatto delle capacità di social CRM sulle performance della relazione con il cliente.

Metodologia: I dati dello studio sono stati raccolti somministrando un questionario on-line a un campione di 90 top manager di imprese italiane e analizzati tramite le equazioni strutturali con approccio PLS.

Risultati: I risultati confermano che: 1) l'orientamento alla relazione con il cliente e l'utilizzo dei social media hanno un impatto positivo e significativo sulle capacità di social CRM sia singolarmente che congiuntamente; 2) le capacità di social CRM hanno un impatto positivo e significativo sulle performance della relazione con il cliente; 3) i social media da soli non hanno un impatto positivo e significativo sulle performance della relazione con il cliente.

Limiti della ricerca: I principali limiti della ricerca riguardano la limitata ampiezza del campione, il non aver testato il modello in differenti settori/classi dimensionali di impresa, e l'aver preso in considerazione solo il punto di vista dei top manager e non quello dei middle manager o dei consumatori.

Implicazioni pratiche: Lo studio suggerisce che i manager dovrebbero sfruttare la grande quantità di informazioni ottenibili dai social media per sviluppare capacità distintive di social CRM e incrementare le performance della relazione con il cliente.

Originalità del paper: Il lavoro rappresenta uno dei primi studi in Italia che esamina l'influenza dei social media sul CRM, il passaggio dal CRM al social CRM e l'impatto delle capacità di social CRM sulle performance della relazione del cliente.

Parole chiave: social media; capacità di social CRM; performance della relazione con il cliente; equazioni strutturali; capacità dinamiche
\end{abstract}

Purpose of the paper: Based on dynamic capabilities theory, the study examines the transformation from CRM to social CRM and the impact of social CRM capabilities on customer relationship performance.

Methodology: An online survey was administered to collect data from a sample of 90 Italian top manager and structural equation was used to test the model.

Findings: Findings show that 1) the customer relationship orientation and social media technology usage have a positive, significant and interactive effect on social CRM capabilities; 2) social CRM capabilities have a positive and significant impact on customer relationship performance; 3) social media technologies do not have a positive and significant impact on customer relationship performance. 
sinergie Vol. 35, N. 103, 2017

Research limits: Main limitations involve: the small size of the sample, the heterogeneity of the observations and the survey sample of top-management team executives.

Practical implications: From a managerial perspective, our findings suggest that companies should utilise social media to take marketing decision, create more effective social CRM capabilities by improving interactions with customers and allowing access to a large number of real customer data, and, consequently, increase customer relationship performance.

Originality of the paper: While extant literature provides a wide analysis of $C R M$, few research has examined how social media technologies interact with CRM, the transformation of CRM to social CRM and how social CRM capabilities enhance customer relationship performance.

Key words: social media; social CRM capabilities; customer relationship performance; structural equation; dynamic capabilities.

\section{Introduzione}

Sin dai primi anni Novanta, il customer relationship management (CRM) ha ricevuto crescente interesse negli studi di marketing (Boulding et al., 2005; Payne e Frow, 2006). Tradizionalmente, il CRM è stato definito come un sistema in grado di gestire i dati derivanti da tutte le fasi della relazione con il cliente (Reinartz et al., 2004). La forte influenza esercitata dalle nuove tecnologie hardware e software ha progressivamente indotto studiosi e professionisti a concepire il CRM come strumento informatico e a trascurarne le implicazioni manageriali e di marketing relazionale. Fino agli anni Novanta, infatti, i sistemi di CRM, hanno mantenuto una natura unidirezionale dall'impresa al cliente, non riuscendo a cogliere gli aspetti relazionali e bidirezionali del rapporto impresa-cliente. A partire dagli anni 2000, l'avvento delle nuove tecnologie, e in particolare delle soluzioni fondate sul Web 2.0 come i social media, hanno creato nuove modalità di interazione cliente-impresa, portando ad un ripensamento e, quindi, ad una ridefinizione del concetto di CRM negli studi di marketing (Malthouse et al., 2013). I social media, favorendo una relazione in tempo reale fra i consumatori e fra essi e le imprese, promuovono un processo di rinnovamento delle modalità di gestione della relazione con il cliente (Cherubini e Pattuglia, 2012; Finotto e Micelli, 2010; Moretti e Tuan, 2014). Da un lato, infatti, i consumatori non devono più essere considerati soggetti esterni all'impresa da cui subiscono passivamente i flussi di comunicazione, ma piuttosto come soggetti interni che, grazie alla grande quantità di informazioni disponibili sui social, condividono giudizi e opinioni sui prodotti, influenzano il processo di acquisto degli altri consumatori, partecipano direttamente alla creazione di un prodotto (Payne e Frow, 2006; Rapp et al., 2010a; Vargo e Lusch, 2004; Verhoef et al., 2010). Dall'altro, le imprese, mediante i social media, possono acquisire grandi quantità di informazioni sui clienti, riuscendo a influenzare in maniera proattiva le loro decisioni. Pertanto, i social media presentano peculiarità uniche per il potenziamento delle capacità del CRM (Malthouse et al., 2013) e per lo sviluppo di soluzioni di relationship marketing. 
L'introduzione dei social media all'interno dei tradizionali sistemi di CRM porta alla formulazione del concetto di social CRM (Choudhury e Harrigan, 2014; Harrigan et al., 2015; Malthouse et al., 2013; Trainor, propri clienti, interagendo direttamente con loro in una logica di scambio bidirezionale e ottimizzando la gestione di ogni fase della relazione con il cliente (Malthouse et al., 2013; Trainor et al., 2014; van Doorn et al., 2010). Il social CRM, infatti, comprende funzioni, capacità e processi che favoriscono il passaggio da una relazione diadica impresa-cliente a una relazione a rete fra impresa e consumatore e fra consumatori stessi (Choudhury, 2014; Greenberg, 2010).

Il social CRM non deve pertanto essere concepito come un mero strumento tecnologico, quanto piuttosto come una nuova capacità distintiva dell'impresa derivante dalla combinazione delle risorse tecnologiche (i social media) con i sistemi di gestione della relazione con il cliente (CRM) che, fornendo al management continue informazioni sui consumatori, permette di incrementare la competitività dell'impresa (Choudhury e Harrigan, 2014). Tuttavia, nonostante la diffusione dei social media e i crescenti investimenti delle imprese nel social CRM, pochi studi hanno analizzato il concetto di social CRM e il suo impatto sulle performance della relazione con il cliente (customer relationship performance, CRP), lasciando molti interrogativi ancora irrisolti.

Il presente lavoro intende quindi contribuire a colmare queste lacune proponendo un modello nel quale la combinazione dei social media con l'orientamento alla relazione con il cliente (customer relationship orientation, CRO) rappresenti il presupposto per la creazione di capacità distintive (capacità di social CRM), il cui fine ultimo sia il miglioramento delle CRP.

In sintesi, lo studio contribuisce all'avanzamento degli studi di management in quanto: 1) è uno dei primi studi, soprattutto a livello italiano, che propone una definizione di capacità di social CRM e ne analizza gli elementi costitutivi; 2) dimostra che le capacità di social CRM sono influenzate dal $\mathrm{CRO}$, dai social media e, soprattutto, dall'effetto congiunto di questi due elementi. In particolare, l'analisi degli effetti congiunti è un tema che ha ricevuto finora poca attenzione negli studi di social media marketing pur rivestendo un ruolo di primaria importanza; 3) analizza l'impatto delle capacità di social CRM sulle CRP.

Il lavoro è organizzato come segue. Dopo aver analizzato i principali contributi della letteratura sul passaggio dal CRM al social CRM, si è proceduto alla formulazione delle ipotesi della ricerca. Successivamente, sono state illustrate la metodologia utilizzata e i principali risultati dello studio. Conclusioni, implicazioni manageriali, limiti e future prospettive di ricerca concludono il lavoro.

\section{Analisi della letteratura}

La teoria delle dynamic capabilitites (DC) rappresenta la base teorica del presente studio. Tale teoria è infatti un perfetto strumento per 
sinergie Vol. 35, N. 103, 2017

esaminare l'impatto delle moderne tecnologie di social media sul CRM, nonché verificarne gli effetti sulle CRP.

La teoria delle DC, prendendo origine dalla teoria della resource based view (RBV), introduce il concetto di capacità. In estrema sintesi, le capacità dinamiche possono essere definite come la capacità di riconfigurare, trasformare, rinnovare le risorse e le competenze chiave possedute dall'impresa (Day, 1994; Eisenhardt e Martin, 2000; Rapp et al., 2010a). Le risorse, da sole, rappresentano infatti solo una materia prima (Ravinchandran e Lertwongsatien, 2005). Lottenimento di performance superiori dipende non solo dal possesso di risorse, come sostenuto dalla $\mathrm{RBV}$, ma dalla capacità dell'impresa di saper combinare, riconfigurare, trasformare e rinnovare tali risorse (Barney, 1991; Day, 1994; Teece et al., 1997).

Numerosi studi (Harrigan et al., 2011; Hooley et al., 2005; Peppard e Ward, 2004; Rai et al., 2006; Roberts e Grover, 2012; Ryals, 2005; Vorhies e Morgan, 2005) hanno utilizzato la teoria delle DC per comprendere in che modo le risorse tecnologiche sono utilizzate dalle imprese per migliorare il CRM e le performance. I risultati di queste ricerche illustrano come le risorse tecnologiche, da sole, non sempre forniscono significativi miglioramenti alle performance. Al contempo, gli studi dimostrano che il miglioramento delle performance si verifica quando tali risorse sono combinate con altre risorse e competenze di impresa. In questo senso, gli ingenti investimenti in risorse tecnologiche, come i social media, a supporto del CRM non portano necessariamente ad un miglioramento delle performance, ma rappresentano le basi per creare e/o rinnovare le capacità organizzative necessarie ad un ottimale utilizzo di tali risorse e a migliorare le performance (Banker et al., 2006). Sono, infatti, frequenti i casi di imprese che pur adottando le stesse risorse tecnologiche, ad esempio i medesimi social media, ottengono risultati talora anche molto diversi. Le risorse social, al tempo stesso, sono facilmente disponibili, sostituibili ed imitabili e, pertanto, da sole non possono contribuire al conseguimento di un duraturo vantaggio competitivo (Thrassou e Vrontis, 2008).

Sulla base di queste brevi considerazioni, la teoria delle DC suggerisce la necessità che le risorse social media siano combinate e integrate con altre risorse e competenze, come il CRM o il CRO, al fine di creare nuove capacità distintive, definibili come capacità di social CRM, in grado di influenzare le CRP. Questo studio, in accordo con Trainor (2014), considera le capacità di social CRM come capacità distintive dell'impresa e le definisce come una combinazione unica delle risorse tecnologiche (i social media) con lorientamento al cliente (CRO) e i tradizionali strumenti di CRM, combinazione che porta a rafforzare la soddisfazione, la fedeltà e la retention del cliente e, quindi, a un miglioramento delle performance della relazione con il cliente.

La Figura 1 mostra il modello concettuale adottato nello studio e le ipotesi di ricerca. Lobiettivo del modello è quello di esaminare dapprima gli elementi costitutivi delle capacità di social CRM e, successivamente, l'influenza di tali capacità sulle performance della relazione con il cliente. 
Fig. 1: Modello e ipotesi dello studio

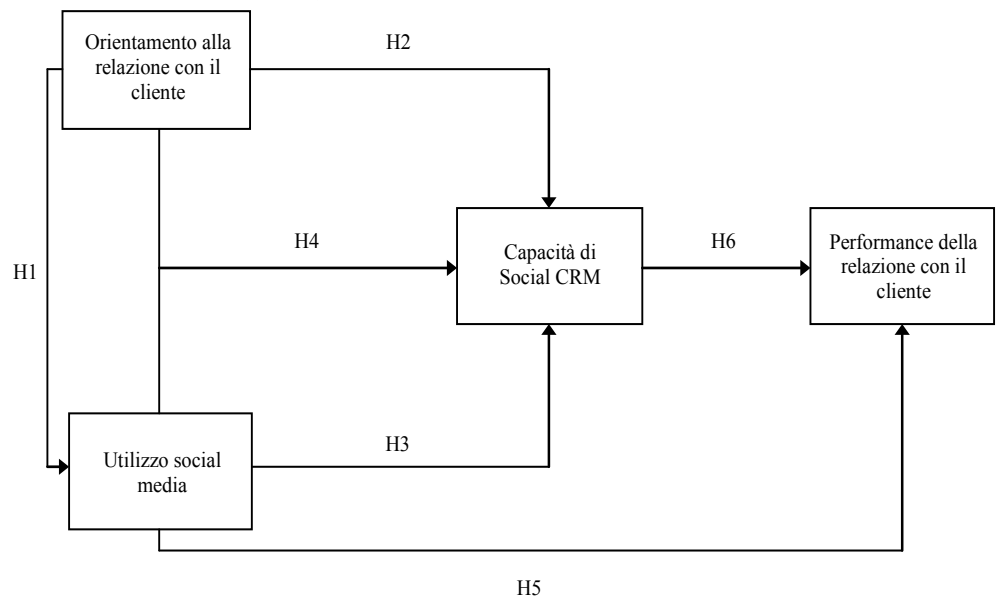

Roberto Chierici Niccolò Gordini Dal CRM al social CRM: uno strumento per incrementarel performance della relazione con il cliente

Fonte: ns. elaborazioni

\section{Ipotesi}

Il concetto di CRO trova fondamento nella teoria di marketing relazionale, secondo la quale un'impresa ottiene e mantiene vantaggio competitivo soddisfacendo gli interessi del consumatore attraverso la creazione di una profittevole relazione di mutuo scambio (Coltman, 2007). Sebbene la necessità di creare stabili e duraturi legami fra l'impresa e il consumatore sia ampiamente condivisa, in letteratura vi sono pochi contributi che analizzano la relazione fra l'utilizzo dei social media e lorientamento ai clienti. La caratteristica principale dei social media è individuabile nella capacità di creare relazioni biunivoche e in tempo reale fra i clienti e tra i clienti e le imprese, incrementando la connettività e la circolazione e diffusione delle informazioni. I social media sono in grado di veicolare le opinioni dei consumatori fra gli utenti e permettono loro di interagire direttamente con le imprese, influenzando i processi decisionali di acquisto e i processi produttivi. In estrema sintesi, date le proprietà relazionali dei social media, è possibile ipotizzare che le imprese che dimostrano un maggior $\mathrm{CRO}$ siano anche quelle più propense all'utilizzo dei social media (USM). Pertanto:

H1: Lorientamento alla relazione con il cliente ha un effetto positivo e significativo sull'utilizzo dei social media

Facendo seguito ad H1, le imprese con un forte CRO mostrano un maggior utilizzo dei social media, una migliore capacità d'interazione con i clienti (Jayachandran et al., 2005) e, conseguentemente, presentano maggiori possibilità di sviluppare capacità di social CRM. Ẽ possibile avanzare alcune considerazioni a sostegno di queste affermazioni. Il CRO permette, anche attraverso l'utilizzo dei social media, di ottenere un crescente numero di informazioni che coadiuvano l'impresa ad 
sinergie Vol. 35, N. 103, 2017

acquisire una più approfondita conoscenza dei consumatori, a interagire e collaborare con loro, nonché a sviluppare risposte tempestive alle esigenze da questi manifestate (Day, 1994). Il CRO incentiva quindi l'impresa a dotarsi di capacità in grado di garantire una efficiente ed efficace gestione di tali informazioni allinterno dell'impresa (Chen e Popovich, 2003; Cooper et al., 2008). Sulla base di queste considerazioni è, pertanto, possibile sostenere che la presenza di un forte $\mathrm{CRO}$ avrà un effetto positivo sullo sviluppo di capacità di social CRM. Conseguentemente:

H2: Lorientamento alla relazione con il cliente ha un effetto positivo e significativo sulle capacità di social CRM

La letteratura ha da sempre considerato la tecnologia un elemento fondante del CRM (Chang et al., 2010; Jayachandran et al., 2005; Rapp et al., 2010a, 2010b). Oggigiorno, tuttavia, questo assunto, non prendendo in considerazione i cambiamenti intervenuti a seguito della diffusione delle nuove tecnologie che facilitano le interazioni sociali, appare riduttivo. In particolare, i social media hanno il potenziale di fornire un accesso semplificato ed in tempo reale ad un gran numero di informazioni sui clienti, sia direttamente, attraverso l'interazione impresa-cliente, sia indirettamente attraverso il monitoraggio dell'interazione cliente-cliente. L'USM ha quindi radicalmente cambiato il modo di gestire il CRM, spronando le imprese a sviluppare nuove capacità per ottimizzare l'utilizzo di una grande quantità di informazioni e migliorare ogni fase della gestione della relazione con il cliente. Pertanto:

H3: L’utilizzo delle tecnologie di social media hanno un impatto positivo e significativo sulle capacità di social CRM

La teoria delle DC afferma la necessità di esaminare gli effetti della complementarietà delle risorse su un determinato fenomeno (Nevo e Wade, 2010; Rapp et al., 2010b; Song et al., 2005; Teece et al., 1997) . In questa prospettiva la complementarietà delle risorse social media con un forte CRO accentuerà gli effetti positivi sulle capacità di social CRM (Malthouse et al., 2013). In conclusione, i social media possono essere concepiti come risorse complementari da utilizzare congiuntamente al $\mathrm{CRO}$ per favorire la creazione di più efficaci ed efficienti capacità di social CRM. Pertanto:

H4: L’utilizzo congiunto dellorientamento alla relazione con il cliente e dei social media influenza positivamente e significativamente le capacità di social CRM

I vantaggi derivanti dall'adozione dei social media hanno incentivato molte imprese a farne ampio utilizzo nel tentativo di incrementare le proprie CRP, misurate, in accordo con Jayachandran et al. (2005), Harrigan et al. (2015), Hooley et al. (2005) e Rapp et al. (2010b), come il livello di soddisfazione e di fedeltà dei propri clienti. Tuttavia, nonostante le imprese adottino spesso i medesimi social media non sempre ottengono gli stessi 
effetti sulle CRP. Le ragioni sottostanti tali differenze possono essere molteplici. Alcuni studi suggeriscono che le cause siano da ricercare nelle modalità di utilizzo dei social media. Più nel dettaglio, l'impiego dei social media ha la possibilità di generare effetti positivi sulle CRP soltanto nel caso in cui questi siano affiancati da competenze in grado di utilizzarne e sfruttarne i punti di forza. La risorsa social media deve, quindi, essere utilizzata in combinazione con altre risorse/competenze come il CRO al fine di creare idonee capacità di social CRM. Altrimenti, il solo utilizzo dei social media, considerati unicamente come strumenti tecnologici in grado di acquisire informazioni sui clienti, può non avere una positiva influenza sulle CRP. Pertanto:

H5: Le tecnologie di social media da sole non hanno un impatto significativo sulle performance della relazione con $i$ client $i$

Le capacità di social CRM, ponendo l'attenzione sull'abilità dell'impresa di interagire in maniera più efficace ed efficiente con i propri clienti, creano i presupposti per una migliore gestione di ogni fase della relazione con il cliente e possono portare ad un miglioramento delle CRP in termini di incremento del tasso di soddisfazione, fedeltà e retention dei clienti. Appare pertanto ragionevole sostenere che il miglioramento delle performance della relazione con il cliente sia l'output naturale di un efficace ed efficiente sistema di capacità di social CRM. Tale affermazione trova riscontro, ad esempio, nel contributo di Rapp et al. (2010b) nel quale si sostiene che le capacità di social CRM determinano la creazione e lo sviluppo di forti relazioni con i clienti che influenzano positivamente la loro soddisfazione e fedeltà, ovvero le performance della relazione con i clienti. Pertanto:

H6: Le capacità di social CRM hanno un impatto positivo e significativo sulle performance della relazione con $i$ clienti

\section{Metodologia}

\subsection{Questionario}

I dati utilizzati nello studio sono stati raccolti somministrando nel 2015 un questionario strutturato online alle posizioni apicali (presidente, amministratore delegato, direttore generale, responsabile marketing) di un campione di 90 imprese italiane. Gli indirizzi mail del campione sono stati ottenuti da IIFAC, società leader in Europea nell'elaborazione di data set di marketing. La scelta di utilizzare lo strumento del questionario online consente alle imprese di rispondere nel rispetto dell'anonimato, in un ambiente user friendly che, non comportando un eccessivo dispendio di tempo, invoglia maggiormente gli intervistati a rispondere rispetto ad altri strumenti tradizionali quali telefono o posta. La scelta, invece, di somministrare il questionario solo alle posizioni apicali si basa su precedenti ricerche (Gordini e Rancati, 2014; 2015; Hambrick e Mason, 1984) le quali sostengono che tali soggetti hanno una più completa visione
Roberto Chierici
Niccolò Gordini

Dal CRM al social

CRM: uno strumento

per incrementare le

performance della relazione

con il cliente 
sinergie Vol. 35, N. 103, 2017

dell'impresa e, pertanto, rappresentano un campione più significativo e rappresentativo per raccogliere le informazioni.

Il questionario, riportato in appendice, si compone di 44 domande, di cui 4 di anagrafica e 40 volte a indagare i 4 costrutti del modello presentati in Figura 1. Ciascun costrutto è stato misurato facendo ricorso ad una serie di items adattati da precedenti studi sul tema (Choudhury e Harrigan, 2014; Harrigan et al., 2015; Jayachandran et al., 2005; Trainor et al., 2014) o elaborati dagli autori dopo una approfondita analisi della letteratura.

I 5 items utilizzati per misurare il CRO sono stati adattati dagli studi di Jayachandran et al. (2005) e di Sin et al., (2005). Questi items servono a comprendere se l'impresa considera la gestione e il mantenimento della relazione con il cliente un asset strategico, se motiva i propri dipendenti ad investire sulla relazione con i clienti e se incentiva l'utilizzo del CRM per l'elaborazione di offerte ad hoc per i clienti.

Riprendendo l'approccio utilizzato nei principali studi sul social media marketing (Jayachandran et al., 2005; Trainor et al., 2014), per comprendere quali sono i social media maggiormente utilizzati dalle imprese (USM) è stato chiesto ai rispondenti di indicare quelli a cui fanno più ampio ricorso fra un elenco di 19 social media. I risultati sono stati successivamente aggregati al fine di ottenere un unico punteggio che esprima quanti e quali strumenti sono usati dalle imprese del campione. Questo approccio, come approfondito nel proseguo del lavoro, può dar adito ad alcuni limiti. Si è tuttavia deciso di ricorrere ad esso perché ampiamente utilizzato in letteratura (Jayachandran et al., 2005; Trainor et al., 2014) e perché esprime in maniera chiara la percentuale di utilizzo da parte delle imprese di ciascun social media.

Le capacità di social CRM sono state misurate facendo ricorso a 31 items ottenuti adattando gli items elaborati dalla letteratura o mediante la creazione di nuovi items sulla base della review della letteratura. L'obiettivo dei 31 items è quello di comprendere se le imprese considerano il social CRM come una combinazione di risorse e competenze (i social media e lorientamento al cliente) che origina nuove capacità distintive le quali incrementano le performance della relazione con il cliente.

Il costrutto CRP, in accordo con la prevalente letteratura sul social CRM (Jayachandran et al., 2005; Hooley et al., 2005; Rapp et al., 2010; Trainor et al., 2014), analizza le performance della relazione con il cliente. In accordo con questi studi, le performance sono definite come il livello di customer satisfaction, customer retention e customer loyalty (Jayachandran et al., 2005; Trainor et al., 2014). Il costrutto valuta, pertanto, il livello con il quale le imprese hanno successo nel soddisfare e fidelizzare i clienti. Il costrutto è stato misurato adattando le scale elaborate da Jayachandran et al. (2005) e Trainor et al. (2014) e si compone di 3 items (tasso di mantenimento, tasso di soddisfazione e tasso di fidelizzazione dei clienti).

Il questionario è stato infine sottoposto ad alcuni test per validare l'unidimensionalità, l'affidabilità e la validità dei costrutti. L'unidimensionalità è stata valutata misurando il loading che i vari items hanno sul proprio costrutto. Se gli items hanno un loading maggiore di 0.5 l'unidimensionalità è confermata (Rivard et al., 1997). I risultati mostrati in Tabella 1 confermano l'unidimensionalità. 
Laffidabilità è stata testata facendo ricorso all'indice alpha di Cronbach (Cronbach, 1951). Tale indice può assumere valori compresi fra 0 e 1 , con valori superiori a 0.6 significativi di un buon livello di coerenza interna e di adeguatezza del test. In questo studio l'indice raggiunge in ogni costrutto un valore superiore a 0.70 a conferma della validità del test. Per avvalorare questo primo risultato è stato inoltre condotto il test composite reliability coefficient (CRC) che deve assumere valori superiori a 0.70 (Fornell e Larcker, 1981). I coefficienti assumono tutti valori superiori a 0.70 , dimostrando l'affidabilità dei 4 costrutti.

La validità è stata, infine, misurata analizzando sia la validità di contenuto (content validity) che la validità di costrutto (construct validity). La validità di contenuto è stata testata somministrando il questionario ad un ristretto numero di manager al fine di migliorarne la struttura prima di somministrarlo al campione analizzato.

La validità di costrutto è stata, invece, misurata analizzando la validità convergente e la validità discriminante. La validità convergente è stata verificata facendo ricorso all'indice average variance extracted (AVE) che deve assumere valori uguali o superiori a 0.5 per dimostrare un buon grado di validità (Fornell e Larcker, 1981; McLure Wasko e Faraj, 2005; Naylor et al., 2012). I nostri risultati dimostrano che tutti e 4 i costrutti soddisfano questo test. La validità discriminante, infine, è stata misurata adottando la procedura di Fornell e Larcker (1981) la quale stabilisce che la validità discriminante di un costrutto esiste quando l'indice AVE del costrutto è maggiore rispetto alla sua varianza condivisa con ogni altro costrutto (Fornell e Larcker, 1981). L'AVE per ciascun costrutto è risultata sempre superiore alla massima varianza condivisa, soddisfacendo pertanto il test.

La Tabella 1 mostra i 4 costrutti utilizzati, il numero di items utilizzato per misurare ciascun costrutto, la fonte bibliografia e i risultati dei test statistici.

Tab. 1: Costrutti del modello e loro proprietà

\begin{tabular}{|c|c|c|c|c|c|c|c|}
\hline Costrutti & $\begin{array}{l}\mathrm{N} . \\
\text { Items }\end{array}$ & $\begin{array}{c}\text { Fonte } \\
\text { Bibliografica }\end{array}$ & $\begin{array}{c}\text { Uni } \\
\text { Dimensionalità } \\
\text { (Loading) }\end{array}$ & $\begin{array}{c}\alpha \mathrm{di} \\
\text { Cronbach }\end{array}$ & CRC & AVE & $\begin{array}{l}\text { Massima } \\
\text { varianza } \\
\text { condivisa }\end{array}$ \\
\hline CRO & 5 & $\begin{array}{l}\text { Harrigan et al. (2015) } \\
\text { Jayachandran et al. (2005); } \\
\text { Sin et al. (2005) }\end{array}$ & 0.73 & 0.82 & 0.81 & 0.72 & $18 \%$ \\
\hline USM & 1 & Nostra Elaborazione & 0.81 & 0.76 & 0.78 & 0.64 & $15 \%$ \\
\hline $\begin{array}{l}\text { Capacità } \\
\text { social } \\
\text { CRM }\end{array}$ & 31 & $\begin{array}{l}\text { Bearden et al. (2011); } \\
\text { Choudhury et al. 2014; } \\
\text { Jayachandran et al. (2005); } \\
\text { Rapp et al. (2010a, b); } \\
\text { Reinartz et al. (2004); } \\
\text { Trainor et al. (2014); } \\
\text { Nostra Elaborazione }\end{array}$ & 0.78 & 0.81 & 0.90 & 0.86 & $15 \%$ \\
\hline CRP & 3 & $\begin{array}{l}\text { Jayachandran et al. (2005); } \\
\text { Hooley et al. (2005); } \\
\text { Rapp et al. (2010b); } \\
\text { Trainor } \text { et al. (2014) }\end{array}$ & 0.75 & 0.74 & 0.83 & 0.71 & $16 \%$ \\
\hline
\end{tabular}

Fonte: ns. elaborazione Roberto Chierici
Niccolò Gordini Dal CRM al social CRM: uno strumento per incrementare le performance della relazione con il cliente 


\section{sinergie}

Vol. 35, N. 103, 2017

\subsection{Data set}

Il questionario così composto è stato somministrato via mail a un campione di 3.152 intervistati. Il tasso di risposta inziale è stato pari all' $1 \%$ (30 imprese). Per incrementare tale tasso di risposta sono state implementate due attività di follow-up. Per ridurre la possibilità di errori di non risposta, come proposto da Kanuk e Berenson (1975), i rispondenti sono stati suddivisi in rispondenti al primo invio e rispondenti al secondo/ terzo invio, per loro stessa natura più simili ai non rispondenti. Il mancato riscontro di differenze significative nei risultati dei due sottogruppi suggerisce che l'errore di non risposta non inficia l'analisi. Il tasso di risposta, dopo le attività di follow-up, è salito al 3\%. Il campione finale è pertanto costituito da 90 imprese prevalentemente di piccole e medie dimensioni ( $\mathrm{N}=49$ pari al $54 \%$ del campione) ed operanti nel settore industria ( $\mathrm{N}=48,53 \%$ del campione) come mostrato in Tabella 2. Questi dati sono in linea con precedenti studi sul tema (Harrigan et al., 2015; Hillebrand et al., 2011).

Tab. 2: Composizione del dataset

\begin{tabular}{|l|c|c|}
\hline \multicolumn{1}{|c|}{ Dimensione } & $\mathrm{N}$ & $\%$ \\
\hline Piccola & 21 & 23 \\
\hline Media & 28 & 31 \\
\hline Grande & 41 & 46 \\
\hline Totale Settore & 90 & 100 \\
\hline \multicolumn{1}{|c|}{$\quad$} & 53 \\
\hline Industria & 48 & 38 \\
\hline Servizi & 34 & 9 \\
\hline Altro & 8 & 100 \\
\hline Totale & 90 & \\
\hline
\end{tabular}

Fonte: ns. elaborazione

\subsection{Metodologia}

In questo studio si è utilizzato un modello di equazioni strutturali (SEM) con approccio PLS (partial least squares). Tale modello risulta particolarmente utile in caso di variabili con un'elevata correlazione, di campioni poco numerosi (Hair et al., 2010) e quando si deve testare un nuovo modello (Gefen et al., 2011) come nel caso in esame. La bontà del modello per avvalorare le ipotesi dello studio è stata testata applicando alcuni indici $\left(\chi^{2}=21.742, \mathrm{p}<0.01\right.$; goodness-of-fit index $=0.968$, adjusted goodness-of-fit index $=0.918$, comparative fit index $=0.91$, root mean square error of approximation $=0.75$ ). I risultati ottenuti confermano la bontà del modello.

La Tabella 3 mostra i risultati del modello, mentre la Figura 2 ne fornisce una rappresentazione grafica. I risultati del modello mostrano che il CRO ha un'influenza positiva e significativa su USM $(\beta=0.28, \mathrm{p}<0.01)$, confermando H1. Inoltre, CRO $(\beta=0.41, \mathrm{p}<0.01)$ e USM $(\beta=0.58, \mathrm{p}<0.05)$ 
hanno entrambe un impatto positivo e significativo sulle capacità di social CRM confermando H2 e H3. Anche l'effetto combinato di CRO e USM ha un impatto positivo e significativo $(\beta=0.61, \mathrm{p}<0.01)$ sulle capacità di social CRM, confermando H4. Infine, i risultati confermano H5 ( $\beta=0.34$, $\mathrm{p}<0.10)$ e H6 $(\beta=0.63, \mathrm{p}<0.01)$. Questo è un importante risultato in quanto conferma che USM da solo non ha un impatto significativo sulle CRP (H5). Al contrario, le capacità di social CRM, derivanti dall'utilizzo combinato di USM con CRO, hanno un effetto positivo e significativo su CRP (H6).

Tab. 3: Risultati del modello SEM-PLS

\begin{tabular}{|c|c|c|c|}
\hline Path & Coefficienti $(\beta)$ & Significatività & Ipotesi \\
\hline CRO $\rightarrow$ USM & 0.28 & 0.01 & H1 - Supportata \\
\hline CRO $\rightarrow$ Capacità di social CRM & 0.41 & 0.01 & H2 - Supportata \\
\hline USM $\rightarrow$ Capacità di social CRM & 0.58 & 0.05 & H3 - Supportata \\
\hline$($ CRO + USM) $\rightarrow$ Capacità di social CRM & 0.61 & 0.01 & H4 - Supportata \\
\hline USM $\rightarrow$ CRP & 0.34 & 0.10 & H5 - Supportata \\
\hline Capacità di social CRM $\rightarrow$ CRP & 0.63 & 0.01 & H6 - Supportata \\
\hline
\end{tabular}

Fonte: ns. elaborazioni

Fig. 2: Rappresentazione grafica dei risultati del modello

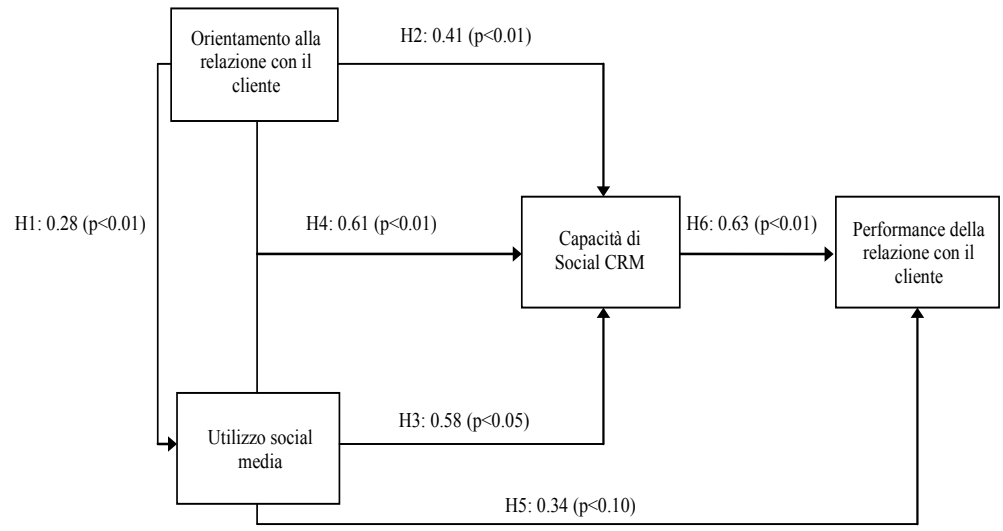

Fonte: ns. elaborazioni

\section{Discussione dei risultati}

Il presente lavoro esamina il ruolo dei social media nella creazione di superiori capacità di social CRM al fine di migliorare la gestione di ogni fase della relazione con il cliente. Il CRM rappresenta infatti un elemento sempre più determinante negli studi di management (Gordini e Veglio, 2014; 2016; Gupta et al., 2006) al quale il recente e repentino sviluppo delle tecnologie di social media ha aggiunto un crescente grado di complessità (Greenberg, 2010; Harrigan et al., 2015; van Doorn et al., 2010).

Lobiettivo dello studio è pertanto quello di dimostrare che i social media e i modelli di CRM sono fortemente interrelati e che il social
Roberto Chierici Niccolò Gordini Dal CRM al social CRM: uno strumento per incrementare le performance della relazione con il cliente 
sinergie Vol. 35, N. 103, 2017

CRM rappresenta un approccio che le imprese dovrebbero adottare per incrementare le CRP.

La prima parte del lavoro analizza gli elementi costitutivi del social CRM. In particolare, $\mathrm{H} 1$ stabilisce che il CRO ha un impatto positivo e significativo sull'USM ed è supportata. Questo è un importante risultato in quanto, confermando precedenti studi (Coltman, 2007; Payne e Frow, 2006), dimostra che non importa quanto siano innovativi e sofisticati i social media, poiché la tecnologia rappresenta solo uno strumento, mentre un radicato orientamento al consumatore rimane l'elemento chiave per adottare innovative soluzioni di CRM (Chen e Ching, 2007; Kohli e Jaworski, 1990; Narver e Slater, 1990).

Lo studio dimostra inoltre che il CRO e l'USM hanno entrambi un'influenza positiva e significativa sulla creazione di distintive capacità di social CRM, sia singolarmente che congiuntamente, confermando $\mathrm{H} 2, \mathrm{H} 3$ e H4. Infatti, da un lato, il forte orientamento al CRO non solo incentiva l'utilizzo dei social media $(\mathrm{H} 1)$, ma anche di un più complesso sistema di CRM in grado di gestire il crescente numero di informazioni ottenibili dall'utilizzo dei social media (H2). Dall'altro, l'utilizzo dei social media facilita l'individuazione, la raccolta e la gestione in tempo reale di un sempre crescente numero di informazioni utili in ogni fase della relazione con il cliente (Baird e Parasnis, 2011; Brodie et al., 2011), confermando H3. La Tabella 4 mostra i social media più utilizzati dalle imprese e quelli che gli intervistati ritengono avranno maggior diffusione nei prossimi tre anni. Da questi primi risultati emerge come le imprese utilizzino contemporaneamente un ampio numero di social media per interagire con i propri clienti. In particolare, i risultati dimostrano che, ad oggi, la maggior parte delle imprese analizzate utilizza Facebook (88\%), Twitter (48\%) e Youtube $(44 \%)$ come principali social media. Nei prossimi tre anni, gli intervistati ritengono che questi social network saranno affiancati anche da social media ad oggi meno diffusi come, ad esempio, Pinterest (dal 2\% al 12\%). Degno di nota, infine, il trend assunto da Facebook che, pur rimanendo il social media più utilizzato, registra una previsione di utilizzo in forte calo (dall' $88 \%$ al 56\%) nei prossimi tre anni.

I risultati dello studio avvalorano inoltre il positivo e significativo effetto congiunto di USM e CRO nello sviluppare capacità di social CRM, confermando H4.

Dopo aver analizzato gli elementi costitutivi di un efficiente sistema di capacità di social CRM, lo studio ha esaminato l'impatto dei social media e delle capacità di social CRM sulle CRP. L'ipotesi 5 investiga se i social media sono in grado da soli di influenzare le CRP. I risultati mostrano che l'utilizzo dei social media, da soli, non ha un impatto significativo sulle CRP. I social media, quindi, non forniscono direttamente un contributo alle CRP ma rappresentano la base su cui costruire e/o rinnovare le capacità distintive dell'impresa necessarie per incrementare tali performance (Banker et al., 2006). Precedenti studi (Hooley et al., 2005; Vorhies e Morgan, 2005) hanno infatti dimostrato che le risorse tecnologiche da sole raramente creano valore per l'impresa e che, al contrario, sono molto più efficienti quando utilizzate in combinazione con altre risorse/competenze (Chang et al., 2010; Jayachandran et al., 2005; Srinivasan e Moorman, 2005). Questi 
risultati suggeriscono quindi che gli investimenti in risorse tecnologiche da soli non necessariamente produrranno un miglioramento dei tassi di mantenimento, soddisfazione e fedeltà del cliente, ossia delle CRP. Tale incremento, invece, si potrà verificare se si creeranno capacità distintive grazie alla combinazione delle risorse tecnologiche con altre risorse di impresa. Sulla base di questo approccio, quindi, il presente studio suggerisce che i social media devono essere combinati con un forte $\mathrm{CRO}$ al fine di creare capacità di social CRM in grado di influenzare le CRP.

Tab. 4: Grado di utilizzo dei social media nel CRM oggi e nei prossimi tre anni

\begin{tabular}{|l|c|c|}
\hline \multicolumn{1}{|c|}{ Social media } & $\begin{array}{c}\text { Percentuale di } \\
\text { utilizzo odierna }\end{array}$ & $\begin{array}{c}\text { Percentuale di utilizzo } \\
\text { nei prossimi tre anni }\end{array}$ \\
\hline Facebook & $88 \%$ & $56 \%$ \\
\hline Google + & $11 \%$ & $11 \%$ \\
\hline Twitter & $48 \%$ & $44 \%$ \\
\hline Linkedin & $24 \%$ & $33 \%$ \\
\hline Xing & $0 \%$ & $0 \%$ \\
\hline Viadeo & $1 \%$ & $2 \%$ \\
\hline Youtube & $44 \%$ & $45 \%$ \\
\hline Dailymotion & $2 \%$ & $4 \%$ \\
\hline Vimeo & $2 \%$ & $5 \%$ \\
\hline Pinterest & $2 \%$ & $12 \%$ \\
\hline Instagram & $27 \%$ & $43 \%$ \\
\hline Flickr & $1 \%$ & $4 \%$ \\
\hline Slideshare & $2 \%$ & $2 \%$ \\
\hline Blog e forum aziendali & $14 \%$ & $16 \%$ \\
\hline Boggling (ad esempio Blogger, Wordpress) & $10 \%$ & $6 \%$ \\
\hline Micro-blogging \& news (Tumblr) & $1 \%$ & $4 \%$ \\
\hline Conference Online/Webinar (Adobe Connect, & $4 \%$ & $8 \%$ \\
\hline Skype, ooVoo) & & $4 \%$ \\
\hline Web Community & $12 \%$ & \\
\hline Altro & $2 \%$ & $17 \%$ \\
\hline
\end{tabular}

Fonte: ns. elaborazioni

Alla luce di queste considerazioni, lo studio ha, infine, investigato se le capacità di social CRM hanno un effetto positivo e significativo sulle CRP, come supposto da H6. I risultati confermano questa ipotesi. Tale risultato è importante perché fornisce nuove evidenze empiriche sulla necessità di utilizzare capacità di social CRM per incrementare le CRP rispetto a precedenti studi che, invece, non avevano fatto registrare una relazione positiva e significativa (Choudhury e Harrigan, 2014; Harrigan et al., 2015) fra l'utilizzo di capacità di social CRM e le CRP.

\section{Conclusioni}

Il CRM rappresenta uno strumento critico nella gestione di impresa, al quale il crescente utilizzo dei social media ha aggiunto una molteplicità di opportunità e minacce (Greenberg, 2010; van Doorn et al., 2010).
Roberto Chierici

Dal CRM al socia

CRM: uno strumento

per incrementare le

performance della relazione

con il cliente 
sinergie Vol. 35, N. 103, 2017

Sulla base dalla teoria delle DC, lo studio ha analizzato l'influenza delle tecnologie di social media sul CRM, la possibilità di adottare un sistema di social CRM e l'impatto di tale sistema sulle CRP.

Per testare le ipotesi della ricerca è stato adottato un modello SEMPLS su un campione di 90 manager di imprese italiane di piccole, medie e grandi dimensioni ed operanti in diversi settori. I risultati del modello confermano l'influenza positiva e significativa degli effetti congiunti dei social media e del CRO sulle capacità di social CRM e di quest'ultime sulle CRP. I risultati confermano, inoltre, che l'utilizzo dei social media, da solo, non ha effetti significativi sulle CRP.

Lo studio contribuisce allavanzamento degli studi di management $\mathrm{e}$ fornisce alcune significative implicazioni manageriali.

Per quanto concerne il contributo agli studi di management, in primo luogo il lavoro propone il concetto di capacità di social CRM, analizzandone gli elementi costitutivi e dimostrando che le capacità di social CRM possono contribuire allincremento delle CRP. In secondo luogo, sulla base delle nostre conoscenze, questo è uno dei pochi studi che esamina nel contesto italiano il concetto di capacità di social CRM e il suo impatto sulle CRP. Infine, in accordo con la teoria delle DC, lo studio introduce l'analisi degli effetti congiunti del CRO e dell'USM sulle capacità di social CRM e sulle CRP. I risultati della ricerca dimostrano che l'effetto positivo e significativo delle capacità di social CRM sulle CRP deriva dalla combinazione delle tecnologie di social media con altre risorse e competenze presenti nell'impresa (ad esempio il CRO). Pertanto, l'utilizzo dei social media, da solo, non è sufficiente a creare un efficiente ed efficace sistema di social CRM e ad incrementare le CRP, risultato ottenibile, invece, mediante l'utilizzo congiunto dei social media con le altre risorse e competenze di impresa.

Da un punto di vista manageriale, invece, se le imprese hanno interesse a sviluppare relazioni di lungo termine con i clienti per consolidare il legame con essi, fidelizzarli e incrementare il tasso di retention, i social media rappresentano strumenti in grado di offrire un prezioso contributo. Tali tecnologie costituiscono, infatti, per gli uomini di marketing, un efficace strumento attraverso il quale interagire con i consumatori e stabilire con essi un'interazione continua e in tempo reale, finalizzata ad acquisire informazioni utili a perfezionare l'offerta dell'impresa e arricchirla con proposte in grado di anticipare o rispondere alle esigenze della clientela incrementando le CRP (Greenberg, 2010; Hennig-Thurau et al., 2010). Pertanto, la sfida per le imprese è quella di monitorare i diversi social media e filtrare la grande quantità di informazioni da essi reperibili. In altri termini, le imprese dovrebbero identificare i social media che i propri clienti utilizzano maggiormente e sviluppare una presenza al loro interno, in modo da monitorare il comportamento dei clienti, comprenderne le esigenze e le opinioni, individuarne le tendenze nei comportamenti d'acquisto per sviluppare un atteggiamento proattivo che permetta da un lato di adattare tempestivamente lofferta ai bisogni del consumatore e, dall'altro, di offrire assistenza e risolvere eventuali problematiche.

Il lavoro presenta infine anche alcuni limiti e futuri percorsi di ricerca. 
In primo luogo lo studio è stato condotto su un campione di limitate dimensioni. Future ricerche dovrebbero quindi testare il modello su un più ampio campione per avvalorarne i risultati.

In secondo luogo, l'analisi è stata svolta su un campione alquanto eterogeneo in termini di settori, classi dimensionali e tipologia di clienti serviti (B2B o B2C). Futuri studi dovrebbero, pertanto, confermare i risultati del modello testandolo su campioni suddivisi per classe dimensionale e/o settore di attività e/o tipologia di clienti serviti (ad esempio B2B o B2C).

In terzo luogo, in linea con precedenti ricerche sul tema (Choudhury e Harrigan, 2014; Harrigan et al., 2015; Rapp et al., 2010; Trainor et al., 2014), il questionario è stato somministrato solo alle posizioni apicali delle imprese. Sebbene molti studi suggeriscano che raccogliere dati dalle posizioni apicali sia utile in virtù dell'elevata conoscenza dell'impresa da parte di questi soggetti, altra letteratura (Baird e Parasnis, 2011) sottolinea lesistenza di significative differenze nella percezione dei social media da parte del top e del middle management d'impresa. Conseguentemente, futuri studi potrebbero investigare come i differenti livelli organizzativi percepiscono l'utilità dei social media nella gestione della relazione con il cliente.

Infine, il contributo analizza l'impatto del social CRM sulle CRP a livello aggregato, senza valutarne gli effetti su ogni fase del ciclo di vita del cliente. Ricerche future potrebbero quindi approfondire i risultati del presente studio analizzando gli effetti dell'utilizzo del social CRM sulle fasi di acquisizione, mantenimento e fidelizzazione del cliente. Tali avanzamenti potrebbero infatti offrire non soltanto un contributo teorico agli studi di management, ma anche aiutare le imprese a comprendere in quale fase della relazione con il cliente le capacità di social CRM risultino di maggiore utilità.

Infine, l'indice adottato per misurare l'utilizzo dei social media fa riferimento solo al numero di strumenti social utilizzati dalle imprese. Sebbene questo metodo di misurazione sia stato ampiamente utilizzato in letteratura, non è tuttavia esente da limiti. Ad esempio, alcuni dei social media analizzati nello studio possono essere più utili per determinate tipologie di imprese (ad esempio imprese B2B piuttosto che B2C). Future ricerche potrebbero proporsi di sviluppare un indice di misurazione dell'utilizzo dei social media che sia in grado di cogliere le differenze fra le imprese sulla base, ad esempio, del settore di attività o che sia in grado di misurare non solo l'utilizzo di una tecnologia, ma anche la sua intensità d'uso. In sintesi, l'adozione di un più robusto indice USM potrebbe fornire una fotografia più chiara di come tali strumenti si legano a differenti capacità e performance.

\section{Bibliografia}

BAIRD C.H., PARASNIS G. (2011), "From social media to social customer relationship management”, Strategy and Leadership, vol. 39, n. 5, pp. 30-37.

BANKER R.D., BARDHAN I.R., CHANG H., LIN S. (2006), "Plant Information Systems, Manufacturing Capabilities, and Plant Performance", MIS Quarterly, vol. 30, n. 2, pp. 315-337.
Roberto Chierici Niccolò Gordini Dal CRM al social CRM: uno strumento performance della relazione con il cliente 
sinergie Vol. 35, N. 103, 2017

BARNEY J. (1991), "Firm resources and sustained competitive advantage", Journal of Management, vol. 17, n. 1, pp. 99-120.

BEARDEN W.O., NETEMEYER R.G., HAWS K.L. (2011), Handbook of marketing scales: multi-item measures for marketing and consumer behavior research, Sage, Los Angeles.

BOULDING W., STAELIN R., EHRET M., JOHNSTON W.J. (2005), "A Customer relationship management Roadmap: What is Known, Potential Pitfallas, and Where to Go", Journal of Marketing, vol. 69, no. 4, pp. 155-166.

BRODIE R.J., HOLLEBEEK L.D., JURIC B., ILIC A. (2011), “Customer Engagement: Conceptual Domain, Fundamental Propositions, and Implications for Research", Journal of Service Research, vol. 14, n. 3, pp. 252-271.

CHANG W., PARK J.E., CHAIY S. (2010), "How does CRM technology transform into organizational performance? A mediating role of marketing capability", Journal of Business Research, vol. 63, n. 8, pp. 849-855.

CHEN I.J., POPOVICH K. (2003), "Understanding customer relationship management (CRM)", Business Process Management Journal, vol. 9, n. 5, pp. 672-688.

CHEN J.S., CHING R.K.H. (2007), "The effects of Information and Communication Technology on Customer relationship management and customer lock-in”, International Journal of Electronic Business, vol. 5, n. 5, pp. 478-498.

CHERUBINI S., PATTUGLIA S. (2012), Social media Marketing. Consumatori, imprese, relazioni, Franco Angeli, Milano.

CHOUDHURY M.M., HARRIGAN P. (2014), "CRM to social CRM: the integration of new technologies into customer relationship management", Journal of Strategic Marketing, vol. 22, n. 2, pp. 149-176.

COLTMAN T. (2007), "Can superior CRM capabilities improve performance in banking", Journal of Financial Services Marketing, vol. 12, n. 2, pp. 102-114.

COOPER M.J., GWIN C.F., WAKEFIELD K.L. (2008), “Cross-functional interface and disruption in CRM projects: Is marketing from Venus and information systems from Mars?", Journal of Business Research, vol. 61, n. 4, pp. 292-299.

CRONBACH L.J. (1951), "Coefficient alpha and the internal structure of tests", Psychometrika, vol. 16, n. 3, pp. 297-334.

DAY G.S. (1994), "The Market-Drive Capabilities Organizations", Journal of Marketing, vol. 58, n. 4, pp. 37-52.

EISENHARDT K.M., MARTIN J.A. (2000), "Dynamic capabilities: what are they?", Strategic Management Journal, vol. 21, n. 10-11, pp. 1105-1121.

FINOTTO V., MICELLI S. (2010), "Web e Made in Italy: la terra di mezzo della comunicazione d'impresa”, Mercati e Competitività, vol. 4, pp. 101-119.

FORNELL C., LARCKER D.F. (1981), "Evaluating Structural Equation Models with Unobservable Variables and Measurement Error", Journal of Marketing Research, vol. 18, n. 1, pp. 39-50.

GEFEN D., STRAUB D.W., RIGDON E.E. (2011), "An Update and Extension to SEM Guidelines for Admnistrative and Social Science Research", MIS Quarterly, vol. 35, n. 2, pp. III-XIV.

GORDINI N., RANCATI E. (2014), Content marketing e creazione di valore Aspetti definitori e metriche di misurazione, G. Giappichelli Editore, Torino.

GORDINI N., RANCATI E. (2015), "Entrepreneurship and growth of small family firms. Evidence from a sample of the artistic craftsmen of Florence", Sinergie Italian Journal of Management, vol. 33, n. 98, pp. 169-194. 
GORDINI N., VEGLIO V. (2014), "Customer relationship management and Data Mining", in Handbook of Research on Novel Soft Computing Intelligent Algorithms (pp. 1-40), IGI Global.

GORDINI N., VEGLIO V., (2016), "Customers churn prediction and marketing Roberto Chierici Niccolò Gordini Dal CRM al social CRM: uno strumento per incrementare le retention strategies. An application of support vector machines based on the AUC parameter-selection technique in B2B e-commerce industry", Industrial Marketing Management, vol. 62, pp. 100-107.

GREENBERG P. (2010), “The impact of CRM 2.0 on customer insight”, Journal of Business and Industrial Marketing, vol. 25, n. 6, pp. 410-419.

GUPTA S., HANSSENS D., HARDIE B., KAHN W., KUMAR V., LIN N., RAVISHANKER N., SRIRAM S. (2006), "Modeling Customer Lifetime Value", Journal of Service Research, vol. 9, n. 2, pp. 139-155.

HAIR J.F., BLACK W.C., BABIN B.J., ANDERSON R.E. (2010), Multivariate Data Analysis, Englewood Cliffs, NJ: Prentice Hall.

HAMBRICK D.C., MASON P.A. (1984), "Upper Echelons: The Organization as a Reflection of Its Top Managers", Academy of Management Review, vol. 9, n. 2, pp. 193-206.

HARRIGAN P., RAMSEY E., IBBOTSON P. (2011), "Critical factors underpinning the e-CRM activities of SMEs", Journal of Marketing Management, vol. 27, n. 5-6, pp. 503-529.

HARRIGAN P., SOUTAR G., CHOUDHURY M.M., LOWE M. (2015), "Modelling CRM in the social media age", Australasian Marketing Journal (AMJ), vol. 23, n. 1, pp. 27-37.

HENNIG-THURAU T., MALTHOUSE E.C., FRIEGE C., GENSLER S., LOBSCHAT L., RANGASWAMY A., SKIERA B. (2010). "The Impact of New Media on Customer Relationships", Journal of Service Research, vol. 13, n. 3, pp. 311-330.

HILLEBRAND B., NIJHOLT J.J., NIJSSEN E.J. (2011), "Exploring CRM effectiveness: an institutional theory perspective", Journal of the Academy of Marketing Science, vol. 39, n. 4, pp. 592-608.

HOOLEY G.J., GREENLEY G.E., CADOGAN J.W., FAHY J. (2005), "The performance impact of marketing resources", Journal of Business Research, vol. 58, n. 1, pp. 18-27.

JAYACHANDRAN S., SHARMA S., KAUFMAN P., RAMAN P. (2005), "The Role of Relational Information Processes and Technology Use in Customer Relationship Management", Journal of Marketing, vol. 69, n. 4, pp. 177-192.

KANUK L., BERENSON C. (1975), "Mail Surveys and Response Rates: A Literature Review”, Journal of Marketing Research, vol. 12, n. 4, pp. 440-453.

KOHLI A., JAWORSKI B. (1990), "Market orientation: the construct, research propositions, and managerial implications", The Journal of Marketing, vol. 54, n. 2, pp. 1-18.

MALTHOUSE E.C., HAENLEIN M., SKIERA B., WEGE E., ZHANG M. (2013), "Managing Customer Relationships in the Social media Era: Introducing the Social CRM House", Journal of Interactive Marketing, vol. 27, n. 4, pp. 270-280.

MCLURE WASKO M., FARAJ S. (2005), "Why Should I Share? Examining Social Capital and Knowledge Contribution in Electronic Networks of Practice", MIS Quarterly, vol. 29, n. 1, pp. 35-57. 
MORETTI A., TUAN A. (2014), "Social media marketing and relationship marketing: revolution or evolution? A first step analysis", Sinergie, n. 93, pp. 115-137.

NARVER J.C., SLATER S.F. (1990), “The Effect of a Market Orientation on Business Profitability", Journal of Marketing, vol. 54, n. October, pp. 20-35.

NAYLOR R.W., LAMBERTON C.P., WEST P.M. (2012), "Beyond the "Like" Button: The Impact of Mere Virtual Presence on Brand Evaluations and Purchase Intentions in Social media Settings", Journal of Marketing, vol. 76, n. 6, pp. 105-120.

NEVO S., WADE, M. (2010), “The formation and value of IT-enabled resources: antecedents and consequences of synergistic relationships", MIS Quarterly, pp. 163-183.

PAYNE A., FROW P. (2005), "A Strategic Framework for Customer Relationship Management”, Journal of Marketing, vol. 69, n. 4, pp. 167-176.

PAYNE A., FROW P. (2006), "Customer Relationship Management: from Strategy to Implementation", Journal of Marketing Management, vol. 22, , pp. 135168.

PEPPARD J., WARD J. (2004), "Beyond strategic information systems: towards an IS capability", The Journal of Strategic Information Systems, vol. 13, n. 2, pp. 167-194.

RAI A., PATNAYAKUNI R., SETH N. (2006), "Firm Performance Impacts of Digitally Enabled Supply Chain Integration Capabilities”, MIS Quarterly, vol. 30, n. 2, pp. 225-246.

RAPP A., AHEARNE M., MATHIEU J., RAPP T. (2010a), "Managing sales teams in a virtual environment", International Journal of Research in Marketing, vol. 27, n. 3, pp. 213-224.

RAPP A., TRAINOR K.J., AGNIHOTRI R. (2010b), "Performance implications of customer-linking capabilities: Examining the complementary role of customer orientation and CRM technology", Journal of Business Research, vol. 63, n. 11, pp. 1229-1236.

RAVINCHANDRAN T., LERTWONGSATIEN C. (2005), "Effect of Information Systems Resources and Capabilities on Firm Performance: A ResourceBased Perspective", Journal of Management Information Systems, vol. 21, n. 4, pp. 237-276.

REINARTZ W., KRAFFT M., HOYER W.D. (2004), "The Customer relationship management Process : Its Relationship Management Measurement and", Journal of Marketing, vol. 41, n. 3, pp. 293-305.

RIVARD S., POIRIER G., RAYMOND L., BERGERON F. (1997), “Development of a measure to assess the quality of user-developed applications", $A C M$ SIGMIS Database, vol. 28, n. 3, pp. 44-58.

ROBERTS N., GROVER V. (2012), "Leveraging information technology infrastructure to facilitate a firm's customer agility and competitive activity: An empirical investigation", Journal of Management Information Systems, vol. 28 , n. 4, pp. 231-270.

RYALS L. (2005), "Making Customer relationship management Work: The Measurement and Profitable Management of Customer Relationships", Journal of Marketing, vol. 69, n. 4, pp. 252-261.

SCHUMACKER R.E., LOMAX R.G. (2010), A Beginner's Guide to Structural Equation Modeling (third), Mahwah, NJ: Lawrence Erlbaum Associates. 
SIN L.Y.M., TSE A.C.B., YIM F.H.K. (2005), “CRM: conceptualization and scale development”, European Journal of Marketing, vol. 39, n. 11/12, pp. 12641290.

SONG M., DROGE C., HANVANICH S., CALANTONE R. (2005), "Marketing Roberto Chierici Dal CRM al social CRM: uno strumento per incrementare le and technology resource complementarity: an analysis of their interaction effect in two environmental contexts", Strategic Management Journal, vol. 26, n. 3, pp. 259-276.

SRINIVASAN R., MOORMAN C. (2005), "Strategic Firm Commitments and Rewards for Customer relationship management in Online Retailing", Journal of Marketing, vol. 69, n. 4, pp. 193-200.

TEECE D.J., PISANO G., SHUEN A. (1997), "Dynamic capabilities and strategic management”, Strategic Management Journal, vol. 18, n. 7, pp. 509-533.

THRASSOU A., VRONTIS D. (2008), "Internet marketing by SMEs: towards enhanced competitiveness and internationalisation of professional services", International Journal of Internet Marketing and Advertising, vol. 4, n. 2/3, pp. 241-261.

TRAINOR K.J. (2012), "Relating Social media Technologies to Performance: A Capabilities-Based Perspective", Journal of Personal Selling and Sales Management, vol. 32, n. 3, pp. 317-331.

TRAINOR K.J., ANDZULIS J., RAPP A., AGNIHOTRI R. (2014), "Social media technology usage and customer relationship performance: A capabilitiesbased examination of social CRM", Journal of Business Research, vol. 67, n. 6, pp. 1201-1208.

VAN DOORN J., LEMON K.N., MITTAL V., NASS S., PICK D., PIRNER P., VERHOEF P.C. (2010), "Customer Engagement Behavior: Theoretical Foundations and Research Directions", Journal of Service Research, vol. 13, n. 3, pp. 253-266.

VARGO S.L., LUSCH R.F. (2004), "Evolving to for Logic Marketing”, The Journal of Marjeting, vol. 68, n. 1, pp. 1-17.

VERHOEF P.C., REINARTZ W.J., KRAFFT M. (2010), "Customer Engagement as a New Perspective in Customer Management", Journal of Service Research, vol. 13, n. 3, pp. 247-252.

VORHIES D.W., MORGAN N.A. (2005), "Benchmarking Marketing Capabilities for Sustainable Competitive Advantage”, Journal of Marketing, vol. 69, n. 1, pp. 80-94. 


\section{sinergie}

Vol. 35, N. 103, 2017

\section{Appendice}

\begin{tabular}{|c|c|c|}
\hline Costrutti e Item & Fonte & $\begin{array}{l}\text { a di } \\
\text { Cronbach }\end{array}$ \\
\hline Orientamento alla relazione con il cliente & & 0.82 \\
\hline $\begin{array}{l}\text { I vostri dipendenti sono incentivati a focalizzarsi sulla } \\
\text { relazione con i clienti }\end{array}$ & $\begin{array}{l}\text { Harrigan et al. (2015); Jayachandran et } \\
\text { al. (2005); Sin et al. (2005) }\end{array}$ & \\
\hline $\begin{array}{l}\text { All'interno della vostra azienda, il mantenimento della } \\
\text { relazione con i clienti è considerata una priorità }\end{array}$ & $\begin{array}{l}\text { Harrigan et al. (2015); Jayachandran et } \\
\text { al. (2005);Sin et al. (2005) }\end{array}$ & \\
\hline $\begin{array}{l}\text { Nella vostra azienda, le relazioni con il clienti sono } \\
\text { considerate come un asset strategico }\end{array}$ & $\begin{array}{l}\text { Harrigan et al. (2015); Jayachandran et } \\
\text { al. (2005); Sin et al. (2005) }\end{array}$ & \\
\hline $\begin{array}{l}\text { La vostra azienda è impegnata nel personalizzare l’offerta } \\
\text { sulla base delle esigenze di specifiche categorie di clienti }\end{array}$ & $\begin{array}{l}\text { Harrigan et al. (2015); Jayachandran et } \\
\text { al. (2005); Sin et al. (2005) }\end{array}$ & \\
\hline $\begin{array}{l}\text { La vostra azienda è impegnata nel personalizzare l'offerta } \\
\text { sulla base delle esigenze del singolo cliente }\end{array}$ & $\begin{array}{l}\text { Harrigan et al. (2015); Jayachandran et } \\
\text { al. (2005); Sin et al. (2005) }\end{array}$ & \\
\hline Utilizzo social media & & 0.76 \\
\hline Facebook & $\begin{array}{l}\text { Choudhury et al., (2014); Harrigan et } \\
\text { al., (2015); Trainor } \text { et al., (2014); } \\
\text { Nostra elaborazione }\end{array}$ & \\
\hline Google + & $\begin{array}{l}\text { Choudhury et al., (2014); Harrigan et } \\
\text { al., (2015); Trainor et al., (2014); } \\
\text { Nostra elaborazione }\end{array}$ & \\
\hline Twitter & $\begin{array}{l}\text { Choudhury et al., (2014); Harrigan et } \\
\text { al., (2015); Trainor } \text { et al., (2014); } \\
\text { Nostra elaborazione }\end{array}$ & \\
\hline Linkedin & $\begin{array}{l}\text { Choudhury et al., (2014); Harrigan et } \\
\text { al., (2015); Trainor } \text { et al., (2014); } \\
\text { Nostra elaborazione }\end{array}$ & \\
\hline Xing & $\begin{array}{l}\text { Choudhury et al., (2014); Harrigan et } \\
\text { al., (2015); Trainor et al., (2014); } \\
\text { Nostra elaborazione }\end{array}$ & \\
\hline Viadeo & $\begin{array}{l}\text { Choudhury et al., (2014); Harrigan et } \\
\text { al., (2015); Trainor et al., (2014); } \\
\text { Nostra elaborazione }\end{array}$ & \\
\hline Youtube & $\begin{array}{l}\text { Choudhury et al., (2014); Harrigan et } \\
\text { al., (2015); Trainor } \text { et al., (2014); } \\
\text { Nostra elaborazione }\end{array}$ & \\
\hline Dailymotion & $\begin{array}{l}\text { Choudhury et al., (2014); Harrigan et } \\
\text { al., (2015); Trainor et al., (2014); } \\
\text { Nostra elaborazione }\end{array}$ & \\
\hline Vimeo & $\begin{array}{l}\text { Choudhury et al., (2014); Harrigan et } \\
\text { al., (2015); Trainor et al., (2014); Nostra } \\
\text { elaborazione }\end{array}$ & \\
\hline Pinterest & $\begin{array}{l}\text { Choudhury et al., (2014); Harrigan et } \\
\text { al., (2015); Trainor et al., (2014);Nostra } \\
\text { elaborazione }\end{array}$ & \\
\hline Instagram & $\begin{array}{l}\text { Choudhury et al., (2014); Harrigan et } \\
\text { al., (2015); Trainor } \text { et al., (2014); Nostra } \\
\text { elaborazione }\end{array}$ & \\
\hline Flickr & $\begin{array}{l}\text { Choudhury et al., (2014); Harrigan et } \\
\text { al., (2015); Trainor } \text { et al., (2014); Nostra } \\
\text { elaborazione }\end{array}$ & \\
\hline Slideshare & $\begin{array}{l}\text { Choudhury et al., (2014); Harrigan et } \\
\text { al., (2015);Trainor et al., (2014); Nostra } \\
\text { elaborazione }\end{array}$ & \\
\hline Blog e forum aziendali & $\begin{array}{l}\text { Choudhury et al., (2014); Harrigan et } \\
\text { al., (2015); Trainor } \text { et al., (2014); Nostra } \\
\text { elaborazione }\end{array}$ & \\
\hline Boggling (ad esempio Blogger, Wordpress) & $\begin{array}{l}\text { Choudhury et al., (2014); Harrigan et } \\
\text { al., (2015); Trainor } \text { et al., (2014); Nostra } \\
\text { elaborazione }\end{array}$ & \\
\hline Micro-blogging \& news (Tumblr) & $\begin{array}{l}\text { Choudhury et al., (2014); Harrigan et } \\
\text { al., (2015); Trainor et al., (2014); Nostra } \\
\text { elaborazione }\end{array}$ & \\
\hline
\end{tabular}

continua ... 
Choudhury et al., (2014); Harrigan et al., (2015); Trainor et al., (2014); Nostra elaborazione

Web Community

Capacità di Social CRM

La vostra azienda ha implementato o utilizza attualmente un sistema e una strategia di CRM?

Utilizza i social media per individuare/rispondere ai cambiamenti del settore (es. concorrenti)

In generale come giudica la qualità del sistema di CRM implementato dalla sua azienda?

Utilizza i social media per veicolare informazioni tra $\mathrm{i}$ diversi dipartimenti della sua azienda?

Se dovesse giudicare l'integrazione tra social media e sistema di CRM della sua azienda, quanto siete soddisfatti?

I social media vi aiutano ad identificare potenziali clienti

I social media alimentano i vostri sistemi di raccolta dati relativi ai clienti potenziali di maggior valore

I social media vi consentono di differenziare la comunicazione in accordo con i diversi target di potenziali clienti

I social media vi aiutano a promuovere una maggiore conoscenza del brand

Grazie ai social media è migliorata l'interazione con i clienti potenziali e la capacità di sviluppare nuove idee

I social media vi aiutano ad interagire con i clienti insoddisfatti e pronti all'abbandono

I social media vi aiutano a ristabilire relazioni con $\mathrm{i}$ clienti inattivi/ persi

I social media vi aiutano a migliorare la vostra capacità di trasformare i prospect in clienti

Le informazioni raccolte attraverso i social media migliorano la capacità dell'azienda di misurare il customer lifetime value

Le informazioni raccolte attraverso i social media migliorano la capacità dell'azienda di misurare la customer retention

Le informazioni raccolte attraverso i social media migliorano la capacità dell'azienda di misurare la customer loyalty

I social media vi aiutano a sviluppare i programmi di fedeltà offerti dall'azienda ai client

I social media vi aiutano a generare e gestire la customer experience

I social media vi aiutano a individuare i vostri attuali clienti più profittevoli

I social media vi aiutano a fidelizzare i vostri clienti più profittevoli

I social media rappresentano un canale fondamentale per il customer care ed il servizio post-vendita della vostra azienda

Le informazioni raccolte attraverso i social media migliorano la capacità di pianificazione delle azioni della forza vendita

Le informazioni raccolte attraverso i social media permettono di supportare l'attività di marketing e branding

Choudhury et al., (2014); Harrigan et al., (2015); Trainor et al., (2014); Nostra elaborazione

Choudhury et al., (2014); Harrigan et al., (2015); Trainor et al., (2014); Nostra elaborazione

Trainor et al. (2014);
Nostra elaborazione

Trainor et al. (2014)

Nostra elaborazione

Trainor et al. (2014)

Nostra elaborazione

(

Bearden et al. (2011); Reinartz et al. (2004)

Bearden et al. (2011); Reinartz et al. (2004)

Bearden et al. (2011); Reinartz et al. (2004)

Choudhury et al. 2014

Bearden et al. (2011); Reinartz et al. (2004)

Bearden et al. (2011); Reinartz et al. (2004)

Bearden et al. (2011); Reinartz et al. (2004)

Bearden et al. (2011); Reinartz et al. (2004)

Choudhury et al. (2014); Jayachandran et al. (2005)

Choudhury et al. (2014); Jayachandran et al. (2005)

Choudhury et al. (2014); Jayachandran et al. (2005)

Bearden et al. (2011); Rapp et al. (2010a, 2010b); Reinartz et al. (2004)

Bearden et al. (2011); Rapp et al. (2010a, 2010b); Reinartz et al. (2004)

Bearden et al. (2011); Rapp et al. (2010a, 2010b); Reinartz et al. (2004)

Bearden et al. (2011); Rapp et al. (2010a, 2010b); Reinartz et al. (2004)

Bearden et al. (2011); Rapp et al. (2010a 2010b); Reinartz et al. (2004)

Choudhury et al. (2014); Jayachandran et al. (2005)

Choudhury et al. (2014); Jayachandran et al. (2005)
Niccolò Gordini

Dal CRM al social

CRM: uno strumento

perin:

performance della relazione

con il cliente

con il cliente

al.

.

\begin{tabular}{l|l} 
& \\
& \\
\hline & \\
\hline & \\
\hline
\end{tabular}

l.

.

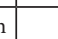

(




\section{sinergie} Vol. 35, N. 103, 2017

... segue

\begin{tabular}{|c|c|c|}
\hline $\begin{array}{l}\text { Grazie ai social media è migliorata l'interazione con i } \\
\text { clienti attuali }\end{array}$ & $\begin{array}{l}\text { Choudhury et al. (2014); Jayachandran } \\
\text { et al. (2005) }\end{array}$ & \\
\hline $\begin{array}{l}\text { I social media vi consentono di personalizzazione } \\
\text { l'offerta sulla base delle esigenze dei clienti }\end{array}$ & Choudhury et al. (2014) & \\
\hline $\begin{array}{l}\text { Attraverso l'interazione con i vostri clienti tramite i social } \\
\text { media, la vostra azienda studia e sviluppa innovazioni di } \\
\text { prodotto/servizio }\end{array}$ & Choudhury et al. (2014) & \\
\hline $\begin{array}{l}\text { I social media vi aiutano a monitorare e gestire i sistemi } \\
\text { di passaparola positivi (customer referral) }\end{array}$ & Reinartz et al. (2004) & \\
\hline $\begin{array}{l}\text { La vostra azienda interviene attivamente nei processi } \\
\text { di customer referral e passaparola all'interno dei canali } \\
\text { social direttamente gestiti da voi (Es. blog aziendale, } \\
\text { forum aziendale, pagina Facebook aziendale...)? }\end{array}$ & $\begin{array}{l}\text { Bearden et al. (2011); Reinartz et al. } \\
\text { (2004) }\end{array}$ & \\
\hline $\begin{array}{l}\text { La vostra azienda interviene attivamente nei processi } \\
\text { di customer referral e passaparola all'interno dei canali } \\
\text { social non direttamente gestiti da voi? }\end{array}$ & $\begin{array}{l}\text { Bearden et al. (2011); Reinartz et al. } \\
(2004)\end{array}$ & \\
\hline $\begin{array}{l}\text { Pensando all'uso che la sua azienda fa dei social media, } \\
\text { lei direbbe che vi aiutano nell'attività di cross-selling con } \\
\text { i vostri clienti }\end{array}$ & $\begin{array}{l}\text { Bearden et al. (2011); Reinartz et al. } \\
\text { (2004) }\end{array}$ & \\
\hline $\begin{array}{l}\text { Pensando all'uso che la sua azienda fa dei social media, } \\
\text { lei direbbe che vi aiutano nellattività di up-selling con i } \\
\text { vostri clienti }\end{array}$ & $\begin{array}{l}\text { Bearden et al. (2011); Reinartz et al. } \\
\text { (2004) }\end{array}$ & \\
\hline Performance della relazione con il cliente & & 0.74 \\
\hline $\begin{array}{l}\text { Quando acquisite nuovi clienti, questi tendono a } \\
\text { rimanere con voi a lungo }\end{array}$ & $\begin{array}{l}\text { Jayachandran et al. (2005); Trainor et } \\
\text { al. (2014) }\end{array}$ & \\
\hline $\begin{array}{l}\text { La vostra azienda ha raggiunto buoni livelli di customer } \\
\text { satisfaction rispetto ai vostri concorrenti }\end{array}$ & $\begin{array}{l}\text { Hooley et al. (2005); Jayachandran et al. } \\
\text { (2005); Rapp et al. (2010b) }\end{array}$ & \\
\hline $\begin{array}{l}\text { In media avete registrato tassi di fedeltà da parte della } \\
\text { vostra clientela superiori a quelli che di solito hanno i } \\
\text { vostri concorrenti }\end{array}$ & $\begin{array}{l}\text { Hooley et al. (2005); Rapp et al. (2010b); } \\
\text { Trainor et al. (2014) }\end{array}$ & \\
\hline
\end{tabular}

Fonte: ns. elaborazioni

\section{Academic or professional position and contacts}

\section{Roberto Chierici}

Research Fellow of Management University Bicocca Milano - Italy e-mail: roberto.chierici@unimib.it

\section{Niccolò Gordini}

Senior Research Consultant

CRIET

e-mail: niccologordini@me.com

sinergie italian journal of management

ISSN 0393-5108 DOI 10.7433/s103.2017.06 pp. $107-128$ 